\title{
RELACIÓN ENTRE LAS PROPIEDADES MECÁNICAS DE SUELOS Y LOS PROCESOS DE GÉNESIS E INTENSIDAD DE USO.
}

\author{
Oscar Seguel $\mathbf{S}^{\mathbf{1}, \mathbf{2}}$, Ivonne Orellana $\mathbf{S}^{\mathbf{1}}$. \\ ${ }^{1}$ Universidad Austral de Chile, Facultad de Ciencias Agrarias, Instituto de Ingeniería Agraria y Suelos. \\ Casilla 567, Valdivia, Chile. \\ ${ }^{2}$ Universidad de Chile, Facultad de Ciencias Agronómicas, Departamento de Ingeniería y Suelos. \\ Casilla 1004, Santiago, Chile. oseguel@uchile.cl
}

\begin{abstract}
Relation between soil mechanical properties and pedogenetic processes and use intensity.
\end{abstract}

Key words: cohesion, preconsolidation, Andisol, Ultisol, friction angle, void ratio.

Soil formation processes and soil management affect its mechanical properties, due to wetting and drying cycles and machinery transit affecting particle arrangement, increasing contact areas and/or disrupting the structure. The relationships between mechanical properties, management and pedological development were evaluated in soils from the south of Chile. Samples from six soils were analyzed, grouped as Andisols and Ultisols, with different management and structural development. Soil samples were equilibrated at $-1,-60$ and $-1600 \mathrm{hPa}$ water tension. Bearing capacity was determined using the confined consolidation test with free drainage, applying loads from 6.25 to $1200 \mathrm{kPa}$. Cohesion and friction angle were determined from direct shearing tests, with normal loads smaller than $50 \mathrm{kPa}$ and a speed of 1 $\mathrm{cm} \mathrm{h}^{-1}$. Mechanical properties of Ultisols were associated with soil management, with structural damage due to intense and long-term use. Preconsolidation value is a mechanical parameter related to soil pedological evolution. Soils were sorted from highest to lowest bearing capacity, associated with lower to higher amounts of precipitation. High mechanical strength is a result of intensified drying processes, resulting in higher values of bulk density in Andisols.

\section{RESUMEN}

Palabras claves: cohesión, preconsolidación, Andisol, Ultisol, ángulo de fricción, relación de vacíos.

Los procesos de formación y el manejo del suelo afectan las propiedades mecánicas de éste, ya que, tanto los ciclos de secado y humectación como el tránsito con maquinaria agrícola, provocan el reordenamiento de las partículas, aumentando las áreas de contacto o desestabilizando las uniones entre éstas. Se evaluó la relación de las propiedades mecánicas con el grado de desarrollo y el manejo de suelos del sur de Chile. Se utilizaron muestras de seis suelos agrupados en dos tipos, Andisoles y Ultisoles, con distinto manejo y desarrollo estructural. Las muestras se equilibraron a tres tensiones mátricas $(-1,-60$ y $-1600 \mathrm{kPa}$ ). Se determinó la capacidad de soporte mediante prueba de consolidación confinada con drenaje libre, con cargas entre 6,25 y $1200 \mathrm{kPa}$. La cohesión y el ángulo de fricción se determinaron mediante pruebas de corte directo con cargas normales inferiores a $50 \mathrm{kPa}$ y velocidad de $1 \mathrm{~cm}$ $\mathrm{h}^{-1}$. Las propiedades mecánicas de los Ultisoles se asocian al manejo, evidenciado por un daño estructural debido al historial de uso más intenso. El valor de preconsolidación resultó ser un parámetro mecánico que se relaciona bien con el grado evolutivo de los suelos, los que se ordenan de mayor a menor capacidad de soporte desde las condiciones climáticas de menor a mayor precipitación. La mayor resistencia mecánica es el resultado de la intensificación de los ciclos de secado, lo que se traduce en mayores valores de densidad aparente en suelos Andisoles. 


\section{INTRODUCCIÓN}

La acción de los factores de formación sobre un material geológico tiene por resultado la expresión de propiedades no sólo morfológicas, sino también físicas, químicas y biológicas del suelo (Buol et al., 1980). Cuando parte de los factores de formación son comunes en su acción, el análisis de la evolución de los suelos se simplifica a los factores más discriminantes, pudiendo estos afectar propiedades aún más específicas.

El manejo del suelo provoca en el corto y mediano plazo cambios en sus propiedades, afectando la funcionalidad de éste. Sin embargo, los sistemas menos intervenidos debiesen tender a un estado de equilibrio que permita asociar las características físico-mecánicas del suelo a sus procesos de formación.

Un suelo se encuentra sometido a tensiones producidas por su propio peso y por la acción de otras fuerzas externas, llegando a un estado de equilibrio. La teoría de la tensión efectiva desarrollada por Bishop señala que, en suelos no saturados, ésta se transmite por las tres fases y queda determinada por:

$$
\mathrm{s}^{\prime}=\left(\mathrm{s}-\mathrm{u}_{\mathrm{a}}\right)+\mathrm{X}\left(\mathrm{u}_{\mathrm{a}}-\mathrm{u}_{\mathrm{w}}\right)
$$

Donde s' es la tensión efectiva sobre la fase sólida, $\mathrm{s}$ la tensión total, $\mathrm{u}_{\mathrm{a}}$ la presión de aire, $\mathrm{X}$ el factor de saturación y $\mathrm{u}_{\mathrm{w}}$ la presión ejercida a través del agua (Fredlund y Rahardjo, 1993). El espacio poroso lleno de aire es el primero en comprimirse cuando existe una continuidad de poros hacia el exterior, por lo que la tensión efectiva se puede simplificar en:

$$
\sqrt{ }^{\prime}=\mathrm{s}-\mathrm{X} »
$$

Donde » es el potencial de presión de agua en los poros, y puede tomar valores negativos (potencial mátrico) o positivos (potencial neumático) bajo la acción de cargas externas. La transmisión de tensiones a través de suelos estructurados es más anisotrópica que en un material geológico homogéneo. El proceso de formación de agregados genera unidades más densas y con un sistema de poros más finos que aquel que se encuentra entre estos agregados. Los poros ubicados entre agregados generarán zonas de menor estabilidad mecánica frente a esfuerzos externos, siendo el potencial mátrico clave, tanto en la generación de unidades estructurales como en la estabilidad de éstas (Horn et al., 1994).

$\mathrm{El}$ factor $\mathrm{X}$ depende del grado de saturación del sistema poroso, de las propiedades hidráulicas y del arreglo de las partículas del suelo. La resistencia del suelo (Ecuación 2) aumenta cuando la disminución del potencial mátrico excede la disminución del factor $\mathrm{X}$, por ende cada suelo tiene una máxima resistencia para un determinado potencial mátrico (Horn et al., 1995). Al contrario, bajo la acción de una carga externa que sobrepase la capacidad de soporte del suelo, se comprime el espacio poroso con aire, el factor $\mathrm{X}$ toma el valor máximo $(\mathrm{X}=1)$ y se generan potenciales neumáticos que disminuyen la resistencia del suelo (Seguel y Horn, 2005).

El estado de las tensiones en el suelo se puede evaluar a partir de la resistencia al corte y la capacidad de soporte. Suelos estructurados poseen una mayor cohesión y ángulo de fricción que suelos homogenizados. Este efecto es más notorio cuando se ha producido un mayor número e intensidad de ciclos de secado y humedecimiento (Horn, 1988). Estos ciclos provocan un mayor acercamiento de las partículas, con el consecuente aumento de los puntos de contacto (Horn y Rostek, 2000). El manejo del suelo, a través de la aplicación de cargas externas, aumenta los puntos de contacto, pero destruye la porosidad secundaria necesaria para el drenaje del agua del suelo.

Como el arreglo de las partículas del suelo y la distribución del tamaño de los poros depende de las propiedades del material parental y los procesos de formación de suelos, debiese existir una relación entre el grado de evolución y las propiedades mecánicas que se utilizan para evaluar el estado de las tensiones del suelo. Así, el objetivo del presente trabajo es analizar la dependencia de las propiedades mecánicas de Andisoles y Ultisoles, que han tenido una baja intervención antrópica, con el grado de desarrollo al interior de cada grupo.

Los suelos dominantes de la zona sur de Chile son Andisoles y Ultisoles (Besoaín, 1985). Los Andisoles chilenos derivan de cenizas volcánicas holocénicas, presentando dataciones de menos de 12.000 años de antigüedad. Poseen 
una alta retención de fósforo ( $>85 \%$ ) y una baja densidad aparente $\left(<0,9 \mathrm{Mg} \mathrm{m}^{-3}\right)$ asociada a una alta porosidad y una fuerte microagregación de formas heterogéneas (Ellies, 1988; 1995). Los Ultisoles de esta zona corresponden a suelos de entre 100.000 y 250.000 años de antigüedad; poseen altos contenidos de arcilla ( $>40 \%)$, abundancia de alófana, producto de la incorporación de cenizas volcánicas, baja saturación de bases $(<30 \%)$ y una mayor densidad aparente $(0,8-1,1$ $\mathrm{Mg} \mathrm{m}^{-3}$ ) que los Andisoles. Ambos tipos de suelos poseen contenidos de materia orgánica mayores al 7\% (Ellies, 1986).

\section{MATERIALES Y MÉTODO}

Se utilizaron muestras no disturbadas de suelos con influencia de cenizas volcánicas. Las muestras fueron colectadas entre los $35 \mathrm{y} 41^{\circ}$ Latitud Sur, con precipitaciones anuales variables entre 500 y $1500 \mathrm{~mm}$ de norte a sur, respectivamente. La ubicación y características de los suelos se presentan en el Cuadro 1.

Para cada uno de estos grupos de suelos se puede identificar un material parental y un relieve en común. Por otra parte, el tiempo de pedogénesis ocurrió principalmente bajo bosque, con formación roble-raulí-coigüe en las áreas de más al norte y bosque siempre verde hacia la décima región (Donoso, 1985). Es así como el clima y el tiempo poseen una mayor relevancia como factores de formación de suelos. A partir de $\operatorname{los} 35^{\circ}$ de latitud Sur no es posible encontrar Ultisoles, por lo que se muestreó un Alfisol con un elevado contenido de arcilla derivada de material volcánico.

Los suelos del Cuadro 1 están ordenados de mayor a menor desarrollo, asociado al tipo y grado estructural y a la condición climática en que se encuentran. Los tres primeros corresponden a suelos desarrollados bajo procesos pedogenéticos más intensos, donde la Serie Mariposa posee el mayor desarrollo estructural, dado el clima en que se desarrolla. La Serie Fresia posee características típicas de los Ultisoles chilenos, mientras que la Serie Metrenco posee las de un paleosuelo, con una fuerte degradación estructural. Para el presente artículo, las Series Mariposa, Fresia y Metrenco serán denominados en forma genérica como suelos Arcillosos. De los tres Andisoles, la Serie Osorno es el concepto central de un suelo Andisol; la Serie Pemehue deriva de cenizas más recientes y su desarrollo estructural es menor; mientras que la Serie Bramadero se desarrolló en una zona con períodos de secado y humectación más intensos que en las otras áreas climáticas.

Se tomaron muestras no alteradas mediante cilindros ( $3 \mathrm{~cm}$ de altura y $7 \mathrm{~cm}$ de diámetro), a dos profundidades $(0-10$ y $10-20 \mathrm{~cm})$ y en dos si-

Cuadro 1. Clasificación taxonómica, ubicación y algunas propiedades importantes de los suelos.

Table 1. Taxonomic classification, location and some important properties of the soils.

\begin{tabular}{|c|c|c|c|c|c|}
\hline \multirow{2}{*}{ Serie } & \multirow{2}{*}{ Clasificación taxonómica ${ }^{\dagger}$} & \multirow{2}{*}{ Ubicación } & \multirow{2}{*}{$\begin{array}{c}\text { Materia } \\
\text { orgánica } \\
(\%)^{*}\end{array}$} & \multicolumn{2}{|c|}{ Arcilla $^{\ddagger}$ Limo $^{\ddagger}$} \\
\hline & & & & 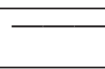 & 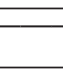 \\
\hline 1. Mariposa & Ochreptic Haploxeralf & $35^{\circ} 50^{\prime} \mathrm{S}$ & 3,8 & 41,7 & 53,1 \\
\hline 2. Fresia & Typic Hapludult & $40^{\circ} 80^{\prime} \mathrm{S}$ & 13,1 & 61,3 & 30,3 \\
\hline 3. Metrenco & Typic Palehumul & $\mathrm{t} 38^{\circ} 70^{\prime} \mathrm{S}$ & 7,54 & 8,0 & 43,5 \\
\hline 4. Bramadero & Humic Haploxerand & $35^{\circ} 50^{\prime} \mathrm{S}$ & 12,2 & 18,7 & 66,9 \\
\hline 5. Osorno & Typic Hapludand & $40^{\circ} 60^{\prime} \mathrm{S}$ & 23,0 & 39,1 & 50,9 \\
\hline 6. Pemehue & Pachic Fulvudand & $38^{\circ} 80^{\prime} \mathrm{S}$ & 18,9 & 22,5 & 67,3 \\
\hline
\end{tabular}

${ }^{\dagger}$ Fuente: USDA (1998).

* Fuente: Mella y Kühne (1985). 
tios para cada suelo, uno bajo vegetación arbórea nativa y otra bajo vegetación pratense.

Las muestras no alteradas fueron equilibradas a tres tensiones mátricas: $-1,-60$ y $-1600 \mathrm{kPa}$ y sometidas a un ensayo de consolidación confinada con drenaje libre (Hartge, 2000) y a una prueba de corte directo (Kézdi, 1980). El ensayo de consolidación se realizó con cargas crecientes de 6,$25 ; 12,5 ; 25 ; 50 ; 100 ; 200 ; 400 ; 800$ y 1200 $\mathrm{kPa}$, con intervalos de 10 minutos entre cargas, midiendo la deformación vertical con un micrómetro electrónico. La prueba de corte se realizó hasta obtener un $20 \%$ de deformación de la muestra; se aplicaron cargas normales menores a la capacidad de soporte del suelo, con el objeto de no dañar la estructura, y a una velocidad de $1 \mathrm{~cm} \mathrm{~h}^{-1}$ para asegurar el drenaje del agua y evitar la generación de presiones neutrales. La capacidad de soporte se estimó a partir de la metodología gráfica propuesta por Casagrande (Dias Junior y Pierce, 1995), mientras que las propiedades de corte se determinaron a partir de la recta de Coulomb (Kézdi, 1980), mediante un ajuste de regresión lineal. El análisis de consolidación consideró tres repeticiones, mientras que la prueba de corte se realizó con la cantidad de repeticiones necesarias para obtener una significancia al $95 \%$.
En forma complementaria se midió la densidad aparente, mediante el método del cilindro, la densidad real, con el método del picnómetro, y el contenido de materia orgánica, mediante combustión húmeda.

\section{RESULTADOS Y DISCUSIÓN}

Propiedades mecánicas y su relación con la evolución y el manejo del suelo.

La Figura 1 presenta los resultados de la capacidad de soporte de los suelos, determinada a partir de la curva de consolidación y para los distintos equilibrios de tensión mátrica.

Para los sitios con bosque nativo (Figuras 1a y 1b), los suelos presentaron un ordenamiento del valor de pre-consolidación en relación al grado de evolución. Entre los suelos Arcillosos, la Serie Mariposa (1) está ubicada más al norte, bajo un régimen climático con eventos mas marcados y extremos de sequía y precipitación. La mayor intensidad de secado genera tensiones internas mayores, lo que se traduce en mayores valores de preconsolidación en un amplio rango de tensión mátrica (Ellies, 1988). La diferencia entre las Series Metrenco y Fresia (2 y 3) radica en que esta última corresponde a un suelo con impor-

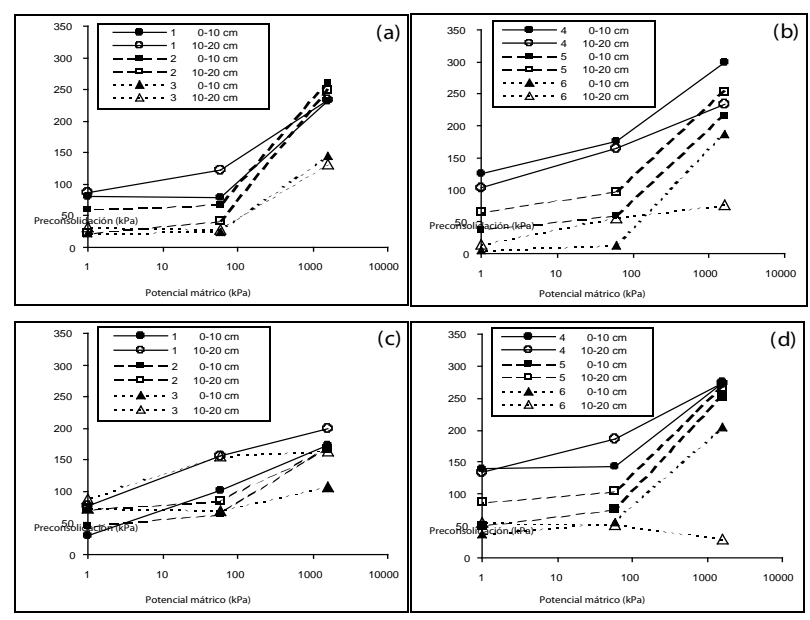

Figura 1. Valor de preconsolidación $(\mathrm{kPa})$ en función de la tensión mátrica para muestras de bosque nativo (a, b) y pradera (c, d). Los números de la leyenda representan el grado de mayor a menor desarrollo según el Cuadro 1.

Figure 1. Loading capacity $(\mathrm{kPa})$ related to matrix tension in forest $(\mathrm{a}, \mathrm{b})$ and prairie $(\mathrm{c}, \mathrm{d})$ samples. Numbers in the legend represent higher to lower evolution degree according to Table 1. 


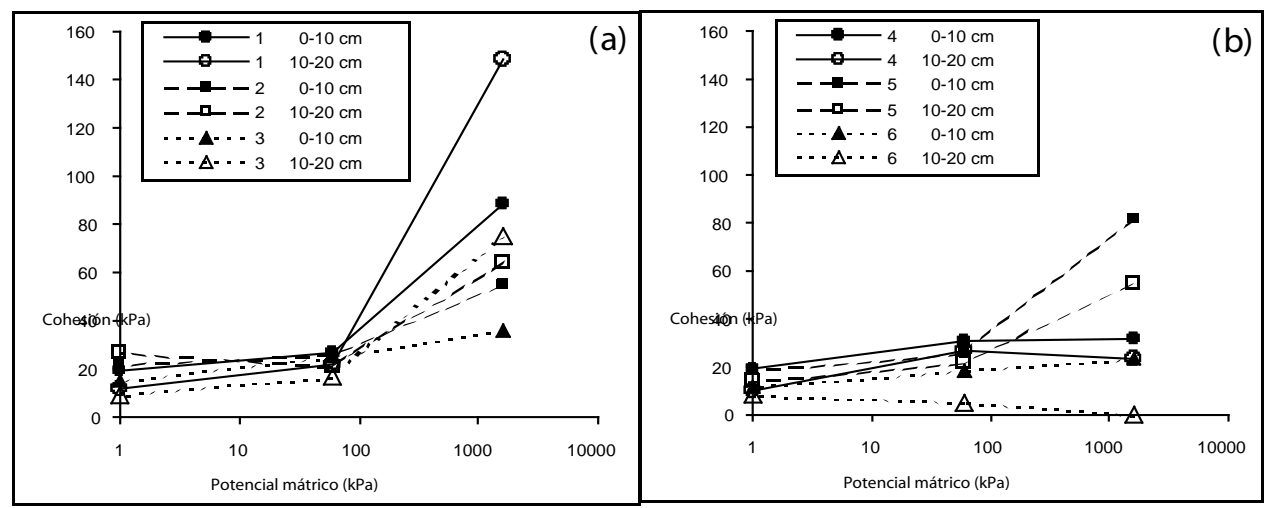

Figura 2. Valores de cohesión en función de la tensión mátrica para los suelos Arcillosos (a) y Andisoles (b) bajo pradera. Los números de la leyenda representan el grado de mayor a menor desarrollo según el Cuadro 1. Figure 2. Cohesion values as a function of matrix tension in (a) Clay soils and (b) Andisols from prairie. Numbers in the legend represent higher to lower evolution degree according to Table 1.

tantes adiciones de material volcánico fresco, lo que le confiere una mayor estabilidad estructural en relación al suelo Metrenco. Por otra parte, el mayor contenido de arcilla de la Serie Fresia (Cuadro 1) le proporciona un mejor desarrollo estructural, con un efecto más claro de los ciclos de secado y humectación. Entre los Andisoles, la Serie Bramadero (4) presentó la mayor capacidad de soporte, ya que por las condiciones climáticas en las que se desarrolla, es el suelo que posee el menor contenido de materia orgánica, la mayor densidad aparente y el mayor número e intensidad de los ciclos de secado.

La tasa de incremento del valor de preconsolidación aumenta en el rango de -60 a $-1600 \mathrm{kPa}$. Sin embargo, cuando los suelos son incorporados para uso ganadero (Figuras 1c y 1d), tanto los valores absolutos como las tasas de incremento son menores, debido al daño sobre la estructura provocado por el tráfico animal, especialmente en los suelos arcillosos (Pietola et al., 2005). La pérdida de capacidad de soporte de la Serie Pemehue, a medida que disminuye el potencial mátrico, se debe a que este suelo no ha sufrido secados tan intensos, por lo que el primer secado a $-1600 \mathrm{kPa}$ provoca que se comporte como un material arenoso, donde la tensión efectiva disminuye por un rápido descenso en el grado de saturación (Seguel et al., 2002).

Los Andisoles, a pesar de poseer un menor contenido de arcilla y de presentar valores de densidad aparente menores que los Ultisoles, tuvieron en promedio un mayor valor de preconsolidación. La cohesión, en cambio, no tuvo una relación clara con el grado de desarrollo del suelo (Figura 2).

Las muestras provenientes de los sectores con bosque nativo presentaron un comportamiento similar a las muestras bajo pradera, por lo que los datos no se presentan. Los suelos Arcillosos (Figura 2), se ordenaron de acuerdo a su grado de desarrollo, y presentaron una mayor cohesión que los Andisoles, asociado a sus texturas más finas. Las muestras de $10-20 \mathrm{~cm}$ presentaron una mayor cohesión que las muestras de 0-10 $\mathrm{cm}$, posiblemente por la mayor presión de confinamiento. En cuanto a los Andisoles (Figura 2), la cohesión no se relacionó con el grado de desarrollo, pero la Serie Pemehue (6) mantuvo un comportamiento concordante con los resultados obtenidos para la capacidad de soporte, ya que las muestras de $10-20 \mathrm{~cm}$ pierden cohesión a medida que el suelo se seca.

Para comparar la condición natural del suelo (bajo bosque nativo) con el manejo bajo pradera, el Cuadro 2 presenta los cambios relativos (en 
Cuadro2. Cambios relativos (\%) de la cohesión y el ángulo de fricción de los suelos. Datos en relación a la condición de bosque. Los valores negativos indican disminuciones.

Table 2. Relative changes (\%) in cohesion and frictional angle of soils. Data related to forest condition. Negative values show decreases.

\begin{tabular}{lccccc}
\hline \multirow{2}{*}{ Scric } & Tensión & \multicolumn{2}{c}{ Cohesión (\%) } & \multicolumn{2}{c}{ Ángulo de fricción (\%) } \\
\cline { 2 - 6 } & $(\mathrm{kPa})$ & $0-10 \mathrm{~cm}$ & $10-20 \mathrm{~cm}$ & $0-10 \mathrm{~cm}$ & $10-20 \mathrm{~cm}$ \\
\hline \multirow{3}{*}{ 1. Mariposa } & -1600 & 184 & 22 & $(-8)$ & $(-4)$ \\
& -60 & 8 & 10 & 22 & $(-25)$ \\
& -1 & 27 & 20 & $(-19)$ & $(-6)$ \\
\cline { 2 - 6 } 2. Fresia & -1600 & 10 & 33 & 7 & $(-16)$ \\
& -60 & 117 & 40 & $(-31)$ & $(-22)$ \\
& -1 & 1000 & 200 & $(-32)$ & $(-42)$ \\
\cline { 2 - 6 } 3. Metrenco & -1600 & 57 & 39 & 74 & 55 \\
& -60 & 150 & $(-19)$ & 3 & 61 \\
& -1 & 67 & $(-40)$ & 24 & 75 \\
4. Bramadero & -1600 & 14 & $(-26)$ & 2 & 12 \\
& -60 & $(-3)$ & 8 & 44 & 0 \\
& -1 & $(-5)$ & $(-60)$ & 17 & 83 \\
\cline { 2 - 6 } 5. Osorno & -1600 & 156 & 41 & 112 & $(-13)$ \\
& -60 & 24 & $(-29)$ & 10 & 39 \\
& -1 & $(-14)$ & $(-46)$ & 48 & 36 \\
\hline \multirow{5}{*}{ 6. Pemehue } & -1600 & $(-8)$ & $(-100)$ & 152 & 12 \\
& -60 & 200 & $(-38)$ & 39 & 27 \\
& -1 & 300 & 14 & $(-26)$ & 20
\end{tabular}

relación a la condición de bosque) para ambos grupos de suelos, tanto de la cohesión como del ángulo de fricción interno.

En los suelos arcillosos, las Series Mariposa y Fresia presentan el comportamiento señalado por Horn (1988), en el sentido que la mayor intensidad de uso destruye los agregados, aumentando la cohesión y disminuyendo el ángulo de fricción. El aumento de la fricción interna en la Serie Metrenco puede deberse a la menor capacidad de soporte de este suelo (Figura 1), lo que en la prueba de corte estaría reflejando el roce entre partículas individuales, principalmente fragmentos gruesos, sobre las cuales la carga normal $\left(\mathrm{s}_{\mathrm{n}}\right)$ genera un acercamiento, con el consecuente aumento de la fricción interna.

En los suelos Andisoles existe una tendencia a disminuir la cohesión, con excepción de las muestras superficiales $(0-10 \mathrm{~cm})$ de los suelos con menor evolución (Osorno y Pemehue). Estos cambios no dependerían de un deterioro estructural, sino de un aumento en la estructura- ción superficial dada por el cambio del sistema radicular de bosque a pradera, esta última mucho más superficial y con carácter fasciculado.

Las disminuciones en profundidad podrían deberse al efecto del pisoteo animal (Pietola et al., 2005). El cambio del sistema vegetacional también explicaría el comportamiento del ángulo de fricción, ya que las raíces fasciculadas generarían agregados de menor tamaño y mayor resistencia que las unidades estructurales mayores. Así, la aplicación de cargas normales durante la prueba de corte provoca un acercamiento de los microagregados, los que se mantienen estables, aumentando el nivel de encaje y puntos de contacto, con el consecuente aumento de la fricción interna (Seguel et al., 2002). En cualquiera de los grupos de suelos, no existe una tendencia clara entre el cambio relativo de las propiedades mecánicas y la tensión de agua al pasar de una condición de bosque nativo al uso bajo pradera.

Al analizar la diferencia de cohesión entre las repeticiones a $-1600 \mathrm{y}-1 \mathrm{kPa}$, se tiene una 


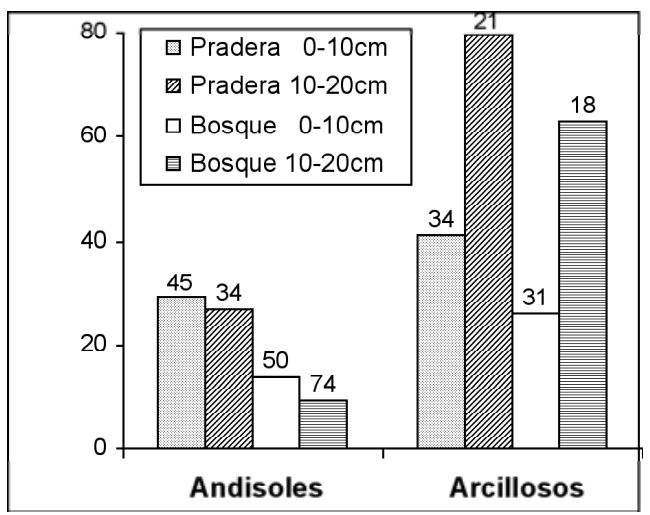

Figura 3. Promedio por grupo de suelo de las diferencias de cohesión entre muestras equilibradas a $-1600 \mathrm{y}-1 \mathrm{kPa}$. Los números sobre las barras indican el porcentaje de cohesión que depende de los agentes agregantes.

Figure 3. Average for group soil of cohesion differences between samples equilibrated to -1600 and $-1 \mathrm{kPa}$. Numbers on bars show cohesion percentage depending on structural agents.

aproximación de la cohesión que depende de las fuerzas generadas por la tensión interna del suelo (contracción de los meniscos de agua) y que no depende de agentes agregantes permanentes. La Figura 3 presenta los resultados promedios según Los valores de cohesión fueron siempre mayores en los suelos arcillosos, pero éstos poseen una mayor dependencia de la atracción de los meniscos de agua, ya que los agentes cementantes aportan menos del 35\% a la cohesión del suelo. Estos resultados tienen relación con la estabilidad al agua, considerablemente menor en los suelos Arcillosos, debido a los menores contenidos de materia orgánica (Ellies et al., 2002). Ambos tipos de suelos poseen una alta CIC y óxidos e hidróxidos de Fe (Besoaín, 1985), por lo que las diferencias de estabilidad se asocian al contenido de materia orgánica dependiente del manejo.

En suelos Andisoles, las diferencias de cohesión en seco y saturado no presentan diferencias entre profundidades, pero sí entre manejos. Esta diferencia, producto del cambio de uso del suelo, estaría provocada por una mayor cohesión dependiente del tránsito sobre el suelo y la intensificación de los procesos de secado y humectación, sin nuevos aportes de materiales cementantes. Para el caso de los suelos Arcillo- sos, existen diferencias tanto entre manejos como entre profundidades. Estos suelos, por su mayor abundancia de partículas finas $<2^{\circ} \mathrm{m}$, desarrollan una mayor cohesión, la que aumenta en profundidad posiblemente por el aumento del contenido de arcilla. En ambos tipos de suelos, el cambio de uso desde el bosque nativo a la pradera provoca la intensificación de los procesos pedogenéticos, y si bien en un comienzo se tiende a homogenizar el suelo, al mediano plazo se intensifican los procesos de secado y humectación, con el consecuente reordenamiento de las partículas y el aumento de la cohesión.

Propiedades mecánicas y su relación con otras propiedades.

Tanto la capacidad de soporte como la cohesión son propiedades que dependen de las características intrínsecas del suelo, de las tensiones internas dadas por el potencial mátrico y de las tensiones externas, dadas por el tipo de carga y el manejo (Horn y Rostek, 2000). En la Figura 4, donde a modo de ejemplo se presentan los datos de las muestras bajo bosque, se puede observar que ambas propiedades poseen una relación lineal; sin embargo, el comportamiento según el tipo de suelo es distinto.

Ambas regresiones de la Figura 4, son estadísticamente significativas $(\mathrm{P} \leq 0,01)$ al ser evaluadas mediante la prueba F. Si bien las correlaciones para las otras profundidades y manejo no son tan satisfactorias como las del bosque, se mantiene la tendencia de una relación rectilínea en los suelos Arcillosos y logarítmica en los suelos Andisoles. El ajuste lineal en los suelos Arcillosos está indicando que, para el rango de cargas analizadas, los aumentos de capacidad de soporte y cohesión mantienen su proporcionalidad, producto de la gran cohesión desarrollada por las tensiones internas generadas en el material arcilloso. Es decir, la mayor capacidad de soporte estaría generada por el acercamiento de las partículas y el aumento de la cohesión. Para el caso de los Andisoles, el manejo (cargas externas) puede generar una mayor capacidad de soporte; sin embargo, en el rango de cargas donde las propiedades mecánicas dependen de la estructura, existe una tendencia a una cohesión máxima limitada por el sistema poroso inter-agregados, el que generaría los planos de falla del suelo. Al seguir aumentando las cargas normales, existiría un aumento de la 


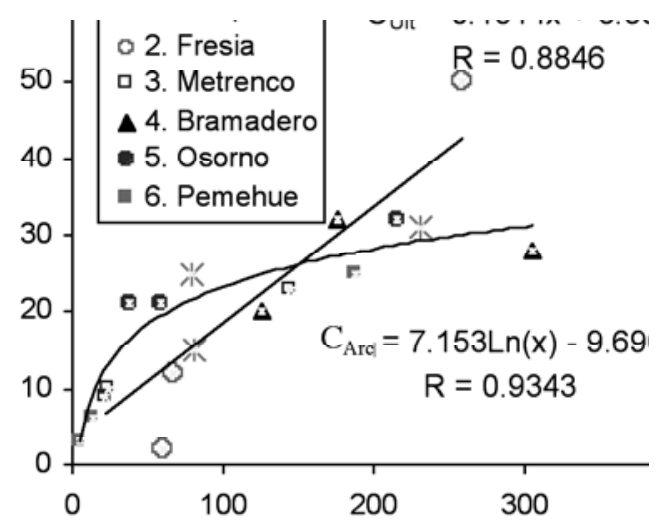

Figura 4. Regresión lineal entre la capacidad de soporte y la cohesión de las muestras de bosque de 0-10 cm ( $\mathrm{C}_{\text {And }}$ : Cohesión Andisoles; $\mathrm{C}_{\text {Arc }}$ : Cohesión Arcillosos).

Figure 4. Lineal regression between loading capacity and cohesion of $0-10 \mathrm{~cm}$ samples collected from forest $\left(\mathrm{C}_{\text {And }}\right.$ : Cohesion Andisols; $\mathrm{C}_{\text {Arc }}$ : Cohesion clayey capacidad de soporte por densificación del suelo, pero no habría un aumento de cohesión debido a la ruptura y deformación de las partículas minerales (Ellies, 1995).

Si se analiza para los Andisoles la relación de las propiedades mecánicas con la densidad aparente, se obtiene un ordenamiento acorde al grado de desarrollo pedológico de los suelos (Figura 5).

Los símbolos de la Figura 5, se agrupan en un orden que dice relación con el grado de evolución, desde el Andisol más reciente (6, Serie Pemehue), con valores de densidad aparente muy bajos, hasta el Andisol de uso agrícola desarrollado más al extremo norte de Chile (4, Serie Bramadero), con un clima más extremo en lo que se refiere a temperatura y ciclos de precipitación y sequía. En cada suelo, los puntos representan los promedios de las repeticiones de dos manejos y dos profundidades.

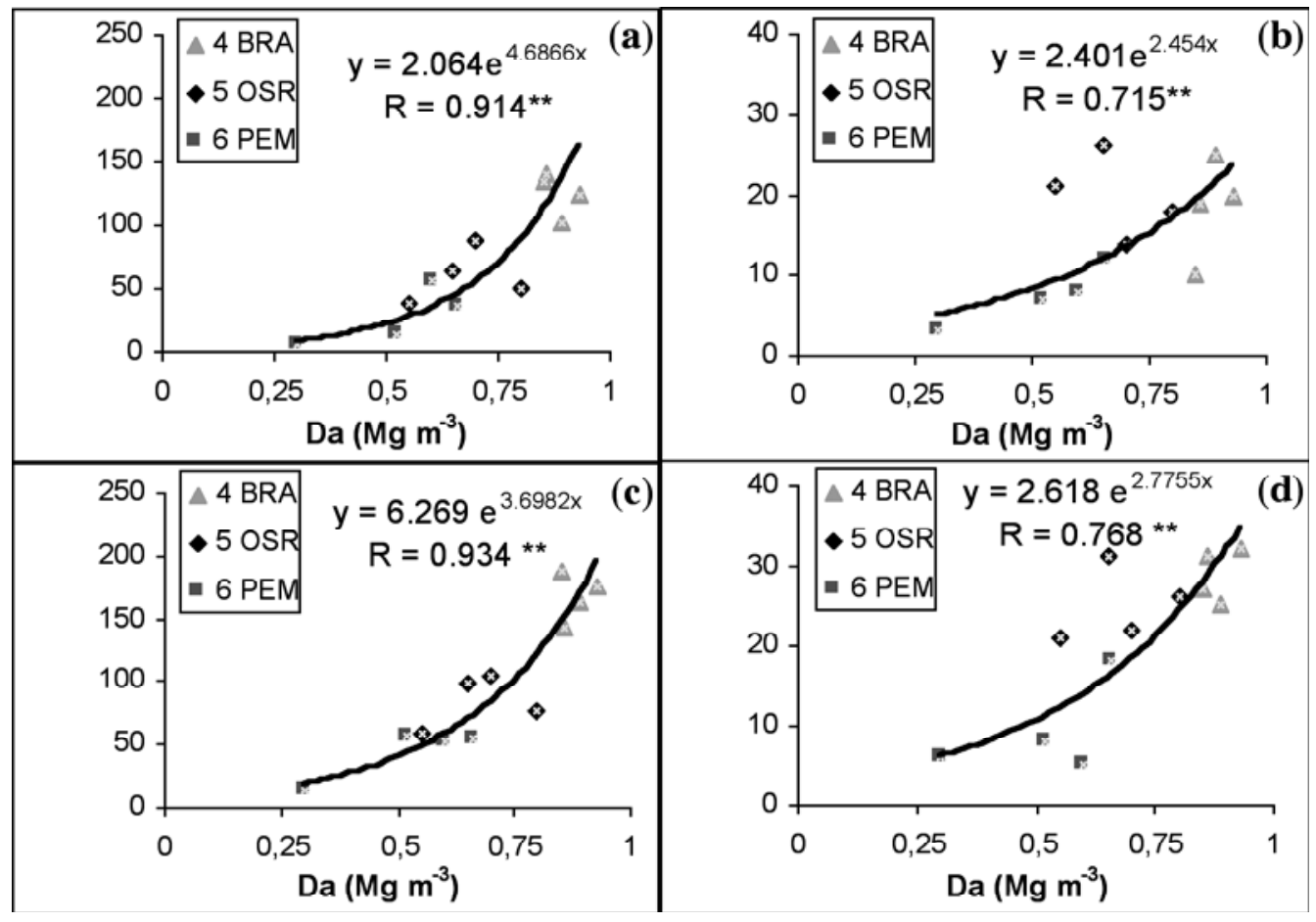

Figura 5. Valores de preconsolidación (a, c) y cohesión (b, d) en función de la densidad aparente (Da, Mg $\mathrm{m}^{-3}$ ) en Andisoles equilibrados a $-1 \mathrm{kPa}(\mathbf{a}, \mathbf{b})$ y $-60 \mathrm{kPa}(\mathbf{c}, \mathbf{d})$. Resultados significativos con $\mathrm{P} \leq 0,01$.

Figure 5. Loading capacity values (a, c) and cohesion (b, d) as functions of bulk density $\left(\mathrm{Da}, \mathrm{Mg} \mathrm{m}^{-3}\right)$ in Andisols equilibrated at $-1 \mathrm{kPa}(\mathbf{a}, \mathbf{b})$ and $-60 \mathrm{kPa}(\mathbf{c}, \mathbf{d})$. Significant results with $\mathrm{P} \leq 0.01$. 


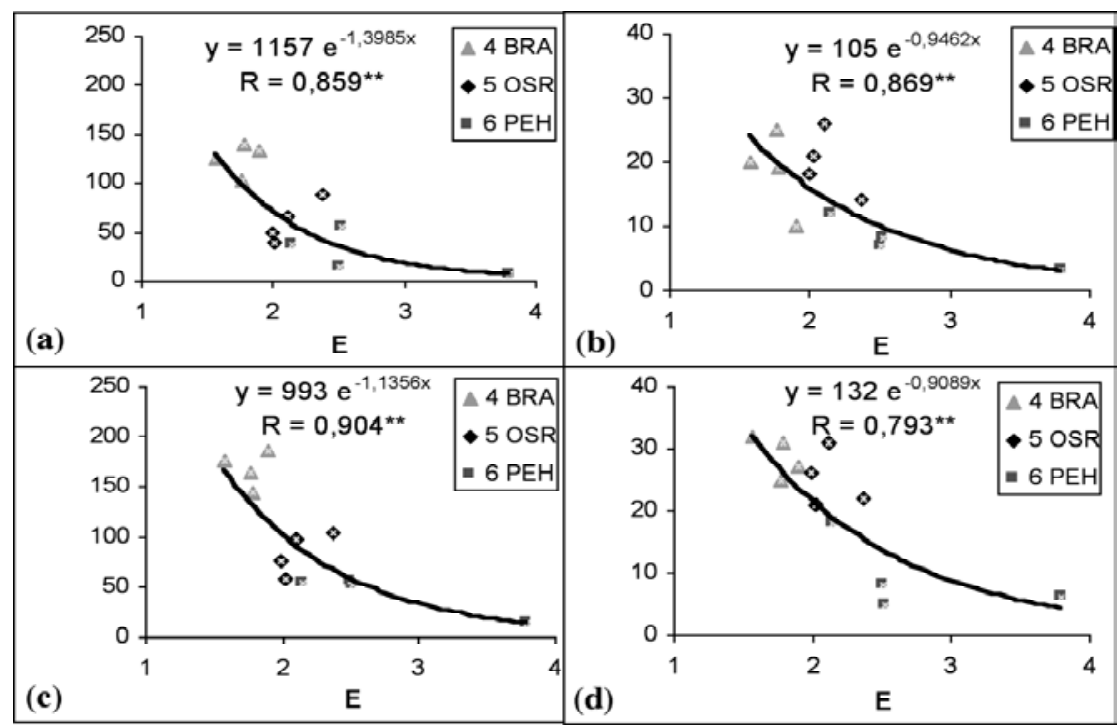

Figura 6.nValores de preconsolidación (a, c) y cohesión $(\mathbf{b}, \mathbf{d})$ en función del número poroso $\left(\mathbf{e}, \mathrm{m}^{3} \mathrm{~m}^{-3}\right)$ en Andisoles equilibrados a tensión mátrica de (a, b) - $1 \mathrm{kPa}$ y (c, d) $-60 \mathrm{kPa}$.

Figure 6. Loading capacity values (a, c) and cohesion (b, d) as functions of pore numbers $\left(\mathbf{e}, \mathrm{m}^{3} \mathrm{~m}^{-3}\right)$ in Andisols equilibrated at matrix tensions of $-1 \mathrm{kPa}(\mathbf{a}, \mathbf{b})$ and $-60 \mathrm{kPa}(\mathbf{c}, \mathbf{d})$.

El grupo de los suelos arcillosos no presentó una buena relación con la densidad aparente, debido a que, por una parte, son suelos más antiguos, con una acumulación de procesos pedológicos que los ha llevado a diferenciarse y, por otra parte, son suelos más frágiles, que dependen en mayor medida del manejo.Además, los resultados físico-mecánicos de estos suelos poseen un mayor grado de dispersión, ya que históricamente han tenido un mayor tiempo e intensidad de uso.

El valor de preconsolidación presentó mejores resultados estadísticos que la cohesión (Figura 5), lo que estaría indicando que las tensiones internas (intensidad y número de ciclos de secado) juegan un rol importante dentro de la génesis del suelo, determinando su capacidad de soporte, mientras que las propiedades de corte dependen en un mayor grado del uso del suelo. La Figura 5 presenta los rangos extremos de densidad aparente de los Andisoles chilenos, por lo que se espera que otros suelos pertenecientes a este Orden se encuentren dentro de la línea de ajuste. Son necesarias nuevas investigaciones para determinar si el valor de preconsolidación se puede constituir como un elemento discriminador de la evolución de suelos Andisoles.

Cuando se analiza la relación de la densidad aparente con los resultados mecánicos de los suelos equilibrados a $-1600 \mathrm{kPa}$, no se obtienen buenas correlaciones, debido a que el secado provoca agrietamiento y planos de falla, lo que genera resultados menos confiables. Tampoco se obtuvieron buenos resultados al correlacionar las propiedades mecánicas con el contenido total de materia orgánica. Es necesario investigar más a fondo para determinar una posible relación con alguna fracción particular o con el contenido de carbono orgánico en los agregados. Como reflejo de la densidad aparente, la porosidad del suelo también tiene una expresión evolutiva del suelo, tal como puede apreciarse en la Figura 6.

Nuevamente existe una clara relación de orden según el grado evolutivo del suelo. A pesar de que el número poroso es una propiedad que se deriva a partir de otras dos medidas en laboratorio (densidad aparente y densidad real), para el caso de la cohesión, la dependencia con el número poroso es más clara que con respecto a la densidad aparente (Figura 5). Resulta necesario evaluar otros Andisoles bajo las condiciones descritas, de manera tal de validar si la capacidad de soporte 
es un buen índice del grado evolutivo de este grupo de suelos.

\section{CONCLUSIONES}

El valor de preconsolidación es una propiedad que se relacionó con la génesis del suelo, ya que los ciclos de secado y humectación provocan el ordenamiento y acercamiento de las partículas, generando un desarrollo estructural que favorece la mayor capacidad de soporte. Así, suelos que acumulan un mayor número e intensidad de ciclos de secado y humectación, presentaron los mayores valores de preconsolidación en un amplio rango de tensiones mátricas e indistintamente del manejo.

El valor de preconsolidación presentó una alta correlación con la densidad aparente de los suelos Andisoles, los que se ordenaron de menor a mayor evolución a medida que aumentó la densidad, en una relación de tipo exponencial.

Las propiedades mecánicas de los suelos Ultisoles se asociaron más con el manejo y la calidad estructural, dada por el historial de uso del suelo.

\section{AGRADECIMIENTOS}

En memoria de Achim Ellies, destacado profesor y amigo.

\section{BIBLIOGRAFÍA}

BESOAÍN, E. 1985. Los Suelos. In: Tosso, J. (ed.). Suelos volcánicos de Chile. INIA-Minagri. Santiago, Chile. pp 23-106.

BUOL, S.; HOLE, F.; McCRACKEN. R. 1980. Soil genesis and classification. USA, The Iowa State University Press. 406 p.

DIAS JUNIOR, M. S.; PIERCE. F. J. 1995. A simple procedure for estimating preconsolidation pressure from soil compresión curves. Soil Technology 8:139-151.

DONOSO, C. 1985. Vegetation synthesis of the Central-Southern Region of Chile. In: Beinroth, F. H.; Luzio, W., Maldonado, F.; Eswaran, H. (eds.). Proceedings of the Sixth International Soil Classification Workshop, Chile and Ecuador. Part II: Tourguide for Chile. Sociedad Chilena de la Ciencia del Suelo, Santiago, Chile. pp:47-53

ELLIES, A. 1986. Efectos de la consolidación mecánica sobre algunos Distrandepts y Palehumults del sur de Chile. Rev. de la Ciencia del Suelo 2:91-97.

ELLIES, A. 1988. Mechanical consolidation in volcanic ash soil. In: Drescher, J.; Horn, R.; De Boodt, M. (eds.). Impact of water and external forces on soil structure. Catena Supplement 11. pp 87-92.

ELLIES, A. 1995. Efecto del manejo sobre las propiedades físicas de suelos trumaos y rojo arcillosos. Bosque 16:101-110.

ELLIES, A., MAC DONALD, R.; RAMIREZ, C. 2002. Efecto de la resistencia a la humectación sobre la estabilidad al agua de los agregados del suelo. R. C. Suelo Nutr. Veg. 2:1-9.

FREDLUND, D. G.; H. RAHARDJO. 1993. Soil mechanics for unsaturated soils. New York, John Wiley \& Sons, Inc.517 p.

HARTGE, K. H. 2000. The effect of soil deformation on physical soil properties. A discourse of the common background. In: Horn, R.; van den Akker J.; Arvidsson, J. (eds.). Subsoil compaction. Distribution, processes and consequences. Advances in Geoecology 32. IUSS. pp: 32-43

HORN, R. 1988. Compressibility of arable land. In: Drescher, J.; Horn, R.; de Boodt, M. (eds.). Impact of water and external forces on soil structure. Catena Supplement 11. pp:53-71

HORN, R.; J. ROSTEK.; 2000. Subsoil compaction processes. In: Horn, R.; van den Akker, J.; Arvidsson, J. (eds.). Subsoil compaction. Distribution, processes and consequences. Advances in Geoecology 32. IUSS. pp: 44-54

HORN, R.; TAUBNER, H.; WUTTKE, M.; BAUMGARTL, T.1994. Soil physical properties related to soil structure. Soil and Tillage Research 30:187216.

HORN, R.; BAUMGARTL, T; KAYSER, R.; BAASCH, S. 1995. Effect of aggregate strength on strength and stress distribution in structured soils. In: Hartge, H.; Stewart. B.A. (eds.). Soil structure its development and function. Advances Soil Science, BocaRaton C.R.C. Pres. pp. 31-52.

KÉZDI, A. 1980. Handbook of soil mechanics. Vol 2. Soil testing. The Netherlands, Amsterdam, Elsevier Publishing Company. 258 p.

MELLA, A.; KÜHNE, A. 1985. Sistemática y descripción de las Familias, Asociaciones y Series de Suelos derivados de materiales piroclásticos de la Zona Central-Sur de Chile. In: Tosso, J. (Ed.). Suelos volcánicos de Chile. INIA-Minagri. Santiago, Chile. pp: 548-716.

PIETOLA, L.; HORN, R.; YLI-HALLA, M. 2005. Effects of trampling by cattle on the hydraulic and mechanical properties of soil. Soil and Tillage 
Research 82: 99-108.

SEGUEL, O.; HORN, R. 2005. Mechanical behavior of a volcanic ash soil (Typic Hapludand) under static and dynamic loading. Soil and Tillage Research 82:109-116.

SEGUEL, O.; ELLIES, A.; MAC DONALD, R.; RAMÍREZ.C. 2002. Capacidad de soporte y resistencia al corte en suelos sometidos a distinto uso. In: Boletín N 18 IX Congreso Nacional de la Ciencia del Suelo. Talca, 4-6 de Noviembre. pp: 47-50

USDA. 1998. Keys to soil taxonomy. Eighth Ed. United Stated Department of Agriculture. Natural Resources Conservation Service. In CD. 